\title{
Neural Processing of Communication Signals: The Extent of Sender-Receiver Matching Varies across Species of Apteronotus
}

\author{
DiDathryne M. Allen and ${ }^{\circledR}$ Gary Marsat
}

https://doi.org/10.1523/ENEURO.0392-18.2019

Department of Biology, West Virginia University, Morgantown, West Virginia 26505

\begin{abstract}
As communication signal properties change, through genetic drift or selective pressure, the sensory systems that receive these signals must also adapt to maintain sensitivity and adaptability in an array of contexts. Shedding light on this process helps us to understand how sensory codes are tailored to specific tasks. In a species of weakly electric fish, Apteronotus albifrons, we examined the unique neurophysiological properties that support the encoding of electrosensory communication signals that the animal encounters in social exchanges. We compare our findings to the known coding properties of the closely related species Apteronotus leptorhynchus to establish how these animals differ in their ability to encode their distinctive communication signals. While there are many similarities between these two species, we found notable differences leading to relatively poor coding of the details of chirp structure occurring on high-frequency background beats. As a result, small differences in chirp properties are poorly resolved by the nervous system. We performed behavioral tests to relate $A$. albifrons chirp coding strategies to its use of chirps during social encounters. Our results suggest that $A$. albifrons does not exchange frequent chirps in a nonbreeding condition, particularly when the beat frequency is high. These findings parallel the mediocre chirp coding accuracy in that they both point to a reduced reliance on frequent and rich exchange of information through chirps during these social interactions. Therefore, our study suggests that neural coding strategies in the CNS vary across species in a way that parallels the behavioral use of the sensory signals.
\end{abstract}

Key words: communication; electrosensory; information; neural coding; sensory tuning

\section{Significance Statement}

Sender-receiver matching is a phenomenon commonly observed in the peripheral nervous system. It enables communication production and reception to evolve together so that conspecifics remain sensitive to important signals. In this article, we examine this phenomenon in the CNS of the weakly electric fish Apteronotus albifrons and compare its processing of communication signals to a closely related species (Apteronotus leptorhynchus). Although some differences across the two species can help to tailor the system for processing species-specific signals, our data indicate that encoding of communication signals in A. albifrons is not as efficient as in A. leptorhynchus for certain categories of signals. Our data support the idea that the extent of sender-receiver matching can vary as a function of behavioral needs.

\section{Introduction}

Sender and receiver matching facilitating communication has been demonstrated across diverse groups, in-

Received October 8, 2018; accepted February 9, 2019; First published March 4, 2019.

K.M.A. and G.M. designed research; K.M.A. performed research; G.M. cluding songbirds (Brumm and Slabbekoorn, 2005), anurans (Schul and Bush, 2002), and insects (Neuhofer et al., 2008). Peripheral tuning and call matching are well

contributed unpublished reagents/analytic tools; K.M.A. analyzed data; K.M.A. and G.M. wrote the paper.

The authors declare no competing financial interests. 
characterized, and studies have shown evidence for sender and receiver matching in response to auditory coding of courtship song (Gerhardt and Schwartz, 2001; Woolley and Moore, 2011; Tootoonian et al., 2012), but little work has been done in other sensory modalities. We aim to identify species-specific variations in the coding properties of central electrosensory neurons and link them to the divergent use of communication signals, showing that the extent of species-specific adaptations results in a variably tight sender-receiver match.

Weakly electric fish are ideal for examining diversification in signal production and reception. Apteronotids share a common mode of communication, the modification of their electric organ discharge (EOD), but exhibit huge variety in signal structure. Voluntary modulations of EOD (chirps) vary dramatically in properties such as duration, frequency, and shape, even between closely related species (Zakon and Smith, 2002; Turner et al., 2007). Likewise, chirp reception is influenced by EOD waveform shape (Petzold et al., 2016), chirp features or structure (Benda et al., 2006; Marsat et al., 2012), and social environment (Stamper et al., 2010). The apteronotid electrosensory system could have coding properties generic enough to process these signals efficiently despite differences in structure and use between species; alternatively, differences in chirp processing could reflect adaptations of the electrosensory system to these differences in chirp production.

Relative EOD frequencies (EODfs) of interacting fish greatly influences chirp perception. These fish perceive ongoing amplitude modulations (AMs; i.e., AM beat) that are the product of two fish with different EOD frequencies interacting at close range (Bastian, 1981). Chirping modulates this beat (Hagedorn and Heiligenberg, 1985; Zupanc and Maler, 1993). In Apteronotus leptorhynchus, low-frequency beats are typical of agnostic encounters between fish of similar sex and size, and fish respond with frequent small chirps ( $<100 \mathrm{~Hz}$ increase; Hupé and Lewis, 2008). Higher frequencies occur between fish of opposite sexes or with large differences in body size and more often elicit big chirps $(>100 \mathrm{~Hz})$, typical of courtship (Hagedorn and Heiligenberg, 1985; Henninger et al., 2018).

Chirp coding has been well characterized in A. leptorhynchus. The two categories of signals described above produce different responses in the primary sensory area, the electrosensory lateral line lobe (ELL). Small chirps on low-frequency beats cause stereotyped bursting among

Funding was provided by West Virginia University and National Science Foundation Grant IOS-1557846.

Acknowledgments: We thank G.T. Smith (Indiana University, Bloomington, IN) for discussions about this project, data on chirp properties, and feedback on the manuscript. We also thank M.J. Chacron (McGill University, Montreal, Canada) for comments on this manuscript.

Correspondence should be addressed to Gary Marsat at gary. marsat@mail.wvu.edu

https://doi.org/10.1523/ENEURO.0392-18.2019

Copyright @ 2019 Allen and Marsat

This is an open-access article distributed under the terms of the Creative Commons Attribution 4.0 International license, which permits unrestricted use, distribution and reproduction in any medium provided that the original work is properly attributed.
ELL pyramidal cells. This encoding strategy, and the structure of the signal itself, means variations in small chirps cannot be discriminated (Marsat et al., 2009; Allen and Marsat, 2018). Conversely, both big and small chirps on high-frequency beats produce heterogeneous responses, and chirp variations are accurately discriminated (Marsat and Maler, 2010; Allen and Marsat, 2018).

The mechanisms of chirp production are similar in Apteronotus albifrons and $A$. leptorhynchus, but chirp structure differs in a number of ways. Chirp duration is the most notable difference in these species. A. leptorhynchus chirps are typically tens of milliseconds long, whereas $A$. albifrons chirps are generally $>100 \mathrm{~ms}$ long (Zupanc and Maler, 1993; Dunlap et al., 1998; Turner et al., 2007). This lengthening is thought to be a recent evolutionary change, with shorter chirps representing the basal state of this branch (Smith et al., 2016). A. albifrons shows differences in frequency tuning from $A$. leptorhynchus (Martinez et al., 2016) that may be adaptations for coding these long chirps. Additionally, A. albifrons chirps typically do not fall into discreet "small" and "big" chirp categories like those of $A$. leptorhynchus (Turner et al., 2007), and chirps of varying durations and frequency are used in all contexts (Kolodziejski et al., 2007). Therefore, it is unknown whether varying chirps have different neural and behavioral impacts in $A$. albifrons.

In this study, we examine the coding of conspecific social signals and their behavioral use to understand whether the sensory system is adapted to the specific characteristics of the communication system of $A$. albifrons, and if so, to what extent. We compare our findings to the behavior and physiology of the closely related $A$. leptorhynchus to identify specific neurophysiological adaptations that reflect differences in the structure and behavioral use of chirps in these two species.

\section{Materials and Methods}

\section{Animals}

All animals were housed according to West Virginia University Institutional Animal Care and Use Committee standards (protocol 151200009.2). Wild-caught $A$. albifrons and $A$. leptorhynchus were obtained from commercial fish suppliers and housed in small groups (1-10 fish/ tank). Tank conductivity was maintained at 200-500 $\mu$ S. Sex was not confirmed, but adult animals with a wide range of EOD frequencies $(600-1300 \mathrm{~Hz})$ were used for all experiments, indicating that we likely had multiple animals of both sexes.

\section{Electrophysiology}

The surgical techniques used were identical to those previously described in the studies by Marsat et al. (2009) and Marsat and Maler (2010). Cells of the lateral segment (LS) of the ELL were targeted. A. albifrons brain anatomy is very similar to that of $A$. leptorhynchus, so major landmark blood vessels described in the study by Maler et al. (1991) and electrode depth served as an adequate guide to locate LS pyramidal cells (see Histology). In vivo recordings were made via metal-filled extracellular electrodes (Frank and Becker, 1964) and amplified with a 
Model 1700 Amplifier (A-M Systems). Data were recorded (Axon Digidata 1500 data acquisition system and AxoScope software, Molecular Devices) at a $20 \mathrm{kHz}$ sampling rate. ON and OFF cells were identified using known response properties, particularly responses to sinusoidal stimulation and spike-triggered average waveforms calculated from responses to $0-60 \mathrm{~Hz}$ noise (Saunders and Bastian, 1984).

\section{Histology}

In $N 9$ fish, correct electrode placement was confirmed by the injection of Dextran Texas Red dye (catalog \#D1829, Thermo Fisher Scientific) at a recording site using double-barreled electrodes, similar to the methods described in the study by Krahe et al. (2008). After recording, the dye was pressure injected with a PicoPump (catalog \#PV820, WPI). Animals were then anesthetized and respirated with a solution of Tricaine-S $(0.5 \mathrm{~g} / \mathrm{L}$; Western Chemicals) and perfused with $4 \%$ paraformaldehyde (catalog \#15712, Electron Microscopy Supply) in 1× PBS. Brains were postfixed overnight, sectioned $(150 \mu \mathrm{m})$, and counterstained with Syto59 nuclear stain (\#S11341, Thermo Fisher Scientific), which allowed for clear distinction between ELL segments. In all marked sections, correct placement within the LS was observed.

\section{Stimuli}

All stimuli were created in MatLab (MathWorks) and sampled at $20 \mathrm{kHz}$. Stimulation was provided by a direct AM of a carrier artificial EOD phase locked to the EOD of the fish rather than by mimicking a second EOD. This method (described below) is commonly used in similar experiments (Benda et al., 2005; Krahe et al., 2008; Marsat et al., 2009) and allows precise control over the stimulus AM. Baseline EOD was recorded via electrodes near the head and tail of the fish. Each EOD cycle triggered a sine-wave generator (catalog \#DG1022A, Rigol) to generate one cycle of a sine wave matched to the sin wave of the animal. This signal was then multiplied using a custom-built signal multiplier (courtesy of the Fortune Laboratory, New Jersey Institute of Technology, Newark, $\mathrm{NJ}$ ) by the AM stimulus to create the desired modulation of the electric field. Stimuli were played through a stimulus isolator (Model 2200, A-M Systems) into the experimental tank via either two $30.5 \mathrm{~cm}$ carbon electrodes arranged parallel to the longitudinal axis of the fish (global stimulation) or two silver chloridized point electrodes $1 \mathrm{~cm}$ apart from each other positioned near the receptive field on the skin of the fish (local stimulation). The stimulus strength was adjusted to provide $\sim 20 \%$ contrast (the difference between the maximum and minimum of amplitude modulation divided by the baseline EOD). For further comments on stimulus strength, see Extended Data Figure 1-1.

Random AM (RAM) stimuli consisted of $30 \mathrm{~s}$ of random noise filtered using either a low pass $(0-20 \mathrm{~Hz})$, band pass $(40-60 \mathrm{~Hz})$, or broadband $(0-60 \mathrm{~Hz})$ Butterworth filter. Each stimulus was played for three repetitions in both global and local stimulation configurations. Sinusoidal AM (SAM) stimuli were 2-s-long stimuli modulated at 2, 5, 15, 30,60 , and $90 \mathrm{~Hz}$ with a $2 \mathrm{~s}$ rest between each frequency,
Table 1: Frequency and duration properties for all chirps used

\begin{tabular}{|c|c|c|c|}
\hline Chirp ID & $\begin{array}{l}\text { Frequency } \\
\text { rise }(\mathrm{Hz})\end{array}$ & $\begin{array}{l}\text { Duration } \\
\text { (ms) }\end{array}$ & Other \\
\hline 1 & 200 & 100 & $\alpha$ Shape \\
\hline 2 & 200 & 200 & $\alpha$ Shape \\
\hline 3 & 350 & 100 & $\alpha$ Shape \\
\hline 4 & 350 & 200 & $\alpha$ Shape \\
\hline 5 & 50 & 50 & $\alpha$ Shape \\
\hline 6 & 100 & 50 & $\alpha$ Shape \\
\hline 7 & 50 & 50 & Antiphase to 5 \\
\hline 8 & 100 & 50 & Antiphase to 6 \\
\hline 9 & 350 & 200 & Two frequency peaks \\
\hline 10 & 350 & 200 & Ramp Shaped \\
\hline 11 & 60 & 10 & A. leptorhynchus small chirp \\
\hline 12 & 122 & 15 & A. leptorhynchus small chirp \\
\hline
\end{tabular}

"Other" indicates differences in chirp shape not due to peak frequency or duration, such as the shape of frequency rise and fall, and the phase of the beat on which the chirp occurred.

repeated at least three times. Step stimulations were 100 -ms-long increases or decreases in amplitude repeated for $30 \mathrm{~s}$.

Chirp stimuli were created by using recorded EOD samples (courtesy of Dr. Troy Smith, Electric Fish Signal Library, http://www.indiana.edu/ efishlab/catalog/faqs. php) to create a $1000 \mathrm{~Hz}$ template for $A$. albifrons EOD shape, thereby accounting for the actual EOD shape and resulting in the AM beat shape. Chirps of varying durations, frequency increases, and frequency rise/fall times (Table 1) were each embedded in this template EOD by decreasing the template EOD period by the inverse of the desired frequency increase for each EOD cycle in the duration of the chirp. For each $1 \mathrm{~Hz}$ of frequency increase, the template amplitude was decreased by $0.11 \%$, based on Dunlap et al. (1998). Chirps were added at the rate of $1 \mathrm{chirp} / \mathrm{s}$. To this chirper EOD, a second EOD, either 990 or $900 \mathrm{~Hz}$, was added to create a combined EOD signal with a beat AM frequency of 10 or $100 \mathrm{~Hz}$. The AM of this stimulus was extracted by rectifying and low-pass filtering the combined EOD signal. The AM signal was delivered during the experiments as described above. Each chirp stimulus was played for at least $30 \mathrm{~s}$ and up to $60 \mathrm{~s}$. Due to time constraints, chirps were only played in the global configuration.

\section{Data analysis}

For all analyses, spike trains were first binarized into a sequence of ones (spike) and zeroes (no spike) using a bin width of $0.5 \mathrm{~ms}$. All analyses described here were performed on these binary sequences in MatLab. Statistical analyses (ANOVA, Student's $t$ test, Wilcoxon rank-sum test) were performed using the MatLab statistical analysis toolbox.

\section{Synchronization coefficient}

The strength of phase locking to SAM stimulation was calculated as follows: 


$$
s=\frac{\sqrt{\left(\sum x_{i}\right)^{2}+\left(\sum y_{i}\right)^{2}}}{n}
$$

where $n$ is the number of spikes in the analysis, and $x$ and $y$ are the cosine and sine of the stimulus phase at which spike $i$ occurs (Goldberg and Brown, 1969; Marsat and Pollack, 2004). The vector strength, $s$, ranges from 0 to 1 , with 1 being perfect precision in responding to a given phase of the cycle.

\section{Coherence}

Lower-bound (stimulus-response) coherence is a measure of linear coding of the stimulus and is calculated by comparing the spike train against the RAM stimulus (Borst and Theunissen, 1999). Stimulus-response ( $\mathrm{S}$ and $\mathrm{R}$ respectively) magnitude-squared coherence $\left(C_{S R}\right)$ was calculated as a function of frequency ( $f$ ) according to the following:

$$
C_{S R}(f)=\frac{\left|P_{S R}(f)\right|^{2}}{P_{S S}(f) P_{R R}(f)}
$$

where $P$ indicates the power-spectral or cross-spectral densities.

Upper-bound (response-response) coherence measures the total information potentially coded in the response, including linear and nonlinear information. It is measured by comparing multiple responses of one neuron to each other to determine response reliability (Borst and Theunissen, 1999). Coherence between responses $R i$ and $R j$ was calculated as follows:

$$
C_{R i R j}(f)=\sqrt{\frac{P_{R i R j}(f) P_{R j i p i}(f)}{P_{R i R i}(f) P_{R j i j j}(f)}}
$$

and averaged across all pairwise combinations of responses.

Envelope responses were determined by computing the lower-bound coherence between the responses and the envelope of the $40-60 \mathrm{~Hz}$ RAM stimuli. To do so, the stimulus $S(t)$ in the lower-bound analysis described above was replaced by the envelope $E(t)$ calculated by rectifying and low-pass filtering the $40-60 \mathrm{~Hz}$ noise stimulus AM. Similar results were obtained when using a Hilbert transform to calculate the envelope signal.

\section{Burst detection}

Bursts in RAM and chirp responses were identified by creating a histogram of all interspike intervals (ISIs) in the response. Bursting neurons have a bimodal, non-Poisson distribution of ISIs, allowing us to visually identify a threshold ISI at the upper boundary of intraburst intervals. Spikes in groups with ISIs below the threshold were classed as occurring in bursts, while all remaining spikes were classed as tonic. This method is similar to that described in the study by Avila-Akerberg et al. (2010)

\section{Chirp detection and discrimination analysis}

Analysis of chirps for detection and discrimination is based on the study by Allen and Marsat (2018). Our analysis accounts for both the firing rate as well as the temporal pattern of spikes to quantify how similar or dissimilar spiking patterns are (van Rossum, 2001). For detection analysis, a window around the chirp $\left[R_{c}(t)\right]$ of length $L(50,105$, or $205 \mathrm{~ms})$ was extracted from the filtered spike train and compared with a window of a beat of the same size $\left[R_{b}(t)\right]$. Different window sizes led to qualitatively similar results, and those using $205 \mathrm{~ms}$ are shown. The responses were convolved with an $\alpha$ filter, $f(t)=t^{*} \exp (-2.45 t / \tau)$, with $\tau$ being the width of the function at half-maximum $(3,10,30$, and $100 \mathrm{~ms} ; 30$ is $\mathrm{ms}$ shown; Machens et al., 2003). Detection accuracy is calculated for population responses by averaging multiple spike trains, as follows:

$$
P R_{i}(t)=\frac{\sum_{i=t}^{n} R_{i}(t)}{n}
$$

The result $\left[P R_{i}(t)\right]$ represents a population of neurons presented with the same stimulus and mimics a neuron integrating postsynaptic potentials with similar weights. Distance $(D)$ was calculated for all sets of combined responses, $P R_{c}(t)$ and $P R_{b}(t)$, creating an array of response distances for each comparison using the following function:

$$
D_{c b}=\frac{\sum_{t=0}^{L}\left[R_{c}(t)-R_{b}(t)\right]^{2}}{L}
$$

The probability distributions of the values in these arrays $\left[P\left(D_{c b}\right)\right.$ or $\left.P\left(D_{b b}\right)\right]$ were used for analysis. Receiver operator characteristic curves were calculated by varying a threshold distance $(T)$ to separate chirp and beat responses. For each threshold, the probability of detection (PD) was calculated as the sum of $P\left(D_{c b}>T\right)$, and the probability of false alarm (PF) as the sum of $P\left(D_{b b}<T\right)$. The error level for each threshold value is $E=\mathrm{PF} / 2+(1-$ $\mathrm{PD} / 2$. The error rate reported in Figure 3 is the minimum value of $E$ across thresholds displayed as a function of the number of spike trains included in the calculation.

Discrimination analyses were similar to those for detection, but rather than comparing chirp and beat responses, the distances between two different chirps were compared. Similar to detection analysis, windows of chirp responses $[R(t)]$ of length $L(50,105$, and $205 \mathrm{~ms})$ were used for analysis, and the results using $205 \mathrm{~ms}$ are shown. The responses were convolved with an $\alpha$ filter, and the population was averaged as described above. Up to 200 random combinations of spike trains from all recorded neurons were used for all comparisons as more become computationally prohibitive without improving results. Responses to a given chirp against a different chirp ( $x$ vs $y$ ) were compared as well as multiple responses to the same chirp ( $x$ vs $x)$. Spike train metric distances $\left(D_{x y}\right.$ or $\left.D_{x x}\right)$ and the ensuing distribution and error rates were calculated as described for the detection analysis. 
A 100 ms Chirp 1

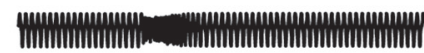

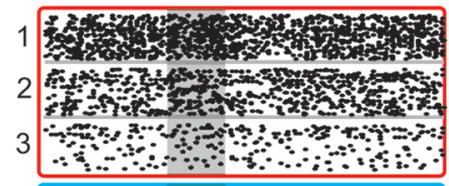

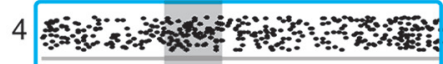

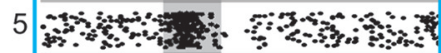

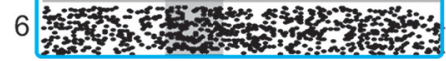

B

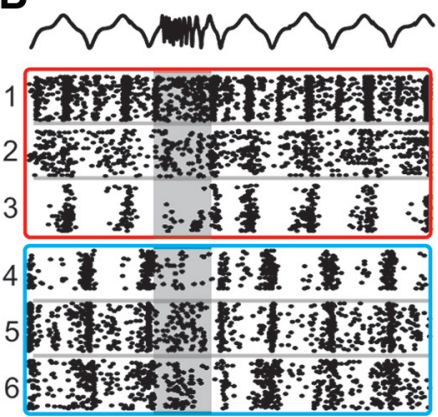

Chirp 4

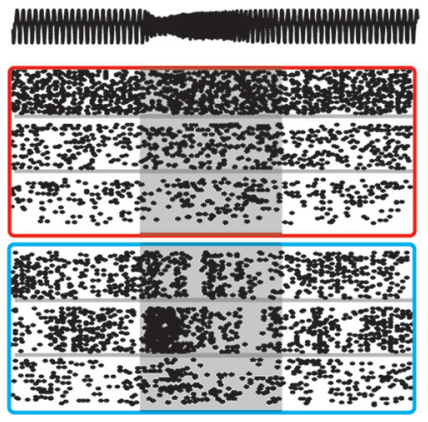

Chirp 7

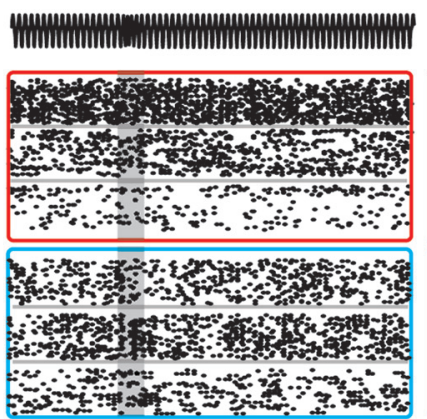

\section{Chirp 9}

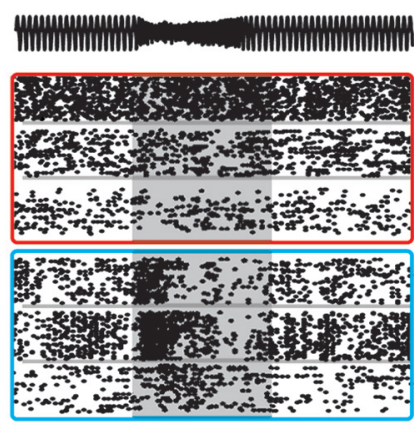

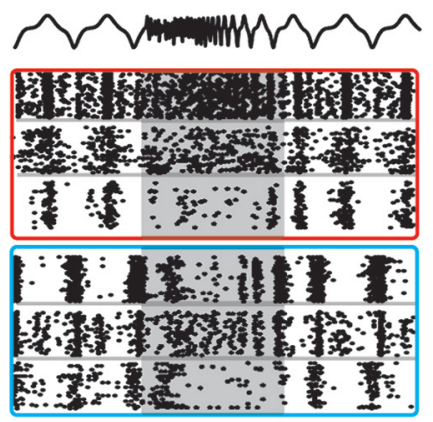

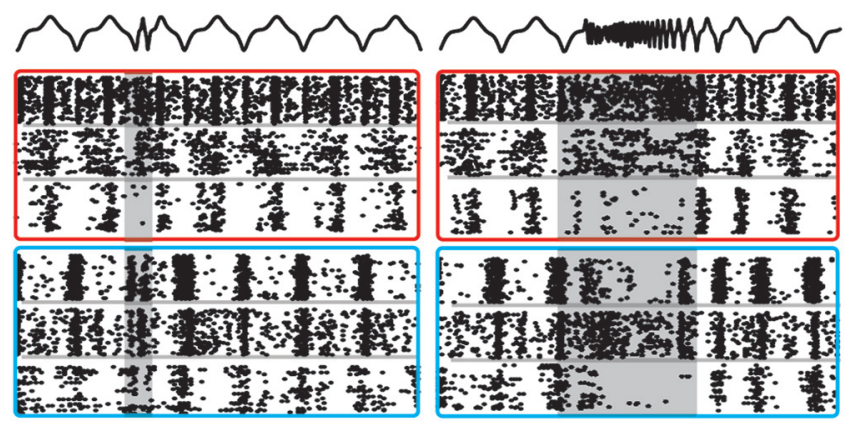

Figure 1. ELL Pyramidal cell responses to conspecific signals. A, Raster plots of chirp responses on a high-frequency (100 $\mathrm{Hz}$ ) beat. AM of the EOD stimulus is shown in black; three representative OFF cells are shown in cyan boxes, while three ON cells responses are displayed in red boxes. The same six neurons are used for all panels. $\boldsymbol{B}$, Responses to chirps on a low-frequency $(10 \mathrm{~Hz})$ beat. For a detailed description of all chirps used, see Table 1. The shaded area highlights the duration of the chirp. See Extended Data Figures 1-1 and 1-2 for comments and analysis of specific response properties (adaptation time and biphasic responses to low frequencies).

\section{Adaptation}

Adaptation to stimuli was measured by using the MatLab Curve Fitting Toolbox to fit an exponential curve to a plot of instantaneous firing rate for each neuron resulting in a time constant $\tau$. The portion of the response to use for fitting was determined by selecting the time of peak firing rate and the following $500 \mathrm{~ms}$.

\section{Behavior}

Twenty-eight behavior trials were conducted in a small tank $(27 \times 27 \times 14 \mathrm{~cm})$ containing water with conductivity, $\mathrm{pH}$, and temperature matched to the home system, and one shelter tube. The tank was enclosed to block ambient light and lit with infrared lights, and all trials were recorded via infrared camera (HD Pro Webcam C920, Logitech). The $14 \mathrm{~cm}$ carbon rod electrodes placed diagonally from each other in each corner of the tank recorded electrical activity, which was then amplified (Model 1700, A-M Systems) and recorded using a computer sound card.

Stranger fish from different home tanks were paired. Defining physical features (size, markings) and EODfs were noted to avoid repeatedly testing the same pairing. Individual fish were used no more than three times, with at least $7 \mathrm{~d}$ between each trial. One fish was selected and allowed to acclimate to the test tank for 20 min before the introduction of the second fish. Recording began imme- diately upon introduction of the intruder fish. Interactions were recorded for $5 \mathrm{~min}$.

To detect chirps, we used a custom MatLab script to create a spectrogram of the electrical recordings to identify individuals and mark chirp times. Chirps were visually identified as $>10 \mathrm{~Hz}$ abrupt frequency increases.

\section{Results}

\section{Diversity in conspecific chirp responses}

In multiple gymnotid species, the LS of the ELL serves as the primary location for the encoding of communication and social signals (Metzner and Juranek, 1997; Metzner, 1999; Marsat et al., 2009); thus, we targeted our recordings to pyramidal cells in that segment. To characterize the pattern of responses of $A$. albifrons pyramidal cells, we played a series of chirps mimicking the natural range of reported $A$. albifrons chirps (Turner et al., 2007). Additionally, for comparison we used a small number of chirps with properties more typical of $A$. leptorhynchus (Zupanc and Maler, 1993; Dunlap et al., 1998). Detailed descriptions of chirp properties used are located in Table 1. In addition to playing chirps with diverse properties, we also varied the frequency of the beat, presenting chirps on both low-frequency $(10 \mathrm{~Hz})$ and high-frequency $(100 \mathrm{~Hz})$ signals.

Figure 1 displays a representative selection of the diversity seen in chirp responses for both ON-type and 
OFF-type pyramidal cells. Qualitatively, responses appear to be heterogeneous with variability among inhibition, excitation, and desynchronization. On high-frequency beats (Fig. 1A), we see a transient increase in tonic firing rate in OFF cells, while ON cells are briefly and weakly inhibited during the chirp. On low-frequency beats (Fig. $1 B$ ), the responses also consisted of brief increases in firing rates, but were additionally characterized by periods of inhibition lasting the entire duration of the chirp. We did not observe synchronized burst firing across the population that is typical of the responses of $A$. leptorhynchus to small chirps on low-frequency beats (Marsat et al., 2009; Marsat and Maler, 2010), the strategy that limits ability to discriminate these chirps (Allen and Marsat, 2018). Therefore, all chirps appear to be coded primarily through increases in firing among OFF cells, and inhibition and desynchronization and cancelation of the beat (Extended Data Fig. 1-1) of ON-cells. These results suggest that $A$. albifrons pyramidal cells use the same coding patterns for all chirp types, but possibly exhibit differences based on beat frequency. Furthermore, a strong adaptation (Extended Data Fig. 1-2) in firing rate seen between the beginning and end of the chirp response could cause chirps of different length to be poorly discriminated. Therefore, we next quantified how well the neural responses could support both the detection and discrimination of chirps.

\section{Detection of chirps varies with beat frequency}

We used a spike metric distance to measure the variability in firing patterns and how reliably an ideal observer could distinguish chirp responses from beat responses. The results are presented as an error level as a function of the number of neural inputs pooled in the analysis. More efficient encoding would lead this measure to drop faster from 0.5 (chance level) as a larger population of neurons is used in the detection task.

A small number of ON cells (about eight) is sufficient to accurately detect chirps comparable to those of $A$. leptorhynchus (Marsat and Maler, 2010; Fig. 2A). However, this level of accuracy only holds for chirps on the $10 \mathrm{~Hz}$ beat. $\mathrm{ON}$-cell performance on $100 \mathrm{~Hz}$ is extremely poor, even with high numbers of spike trains included in the analysis (Fig. 2B). OFF cells are also able to detect chirp occurrence on low-frequency beats for all chirp types (Fig. 2C), but on $100 \mathrm{~Hz}$ detecting chirps was less reliable and accurate (Fig. 2D). The data suggest that with a large enough sample of neurons accurate detection is possible, but less robustly than on low frequencies. Therefore, while chirps in all contexts are potentially detected, detection sensitivity is much higher in lowfrequency contexts.

\section{Discrimination between chirps also varies}

We assessed the amount of information carried by the response pattern about chirp properties that could support the discrimination of chirp variants. This analysis is similar to that used for chirp detection, but, rather than comparing responses of chirps to beats, we compare responses between chirps. While $A$. albifrons chirps are not typically grouped into distinct categories, we still use
A
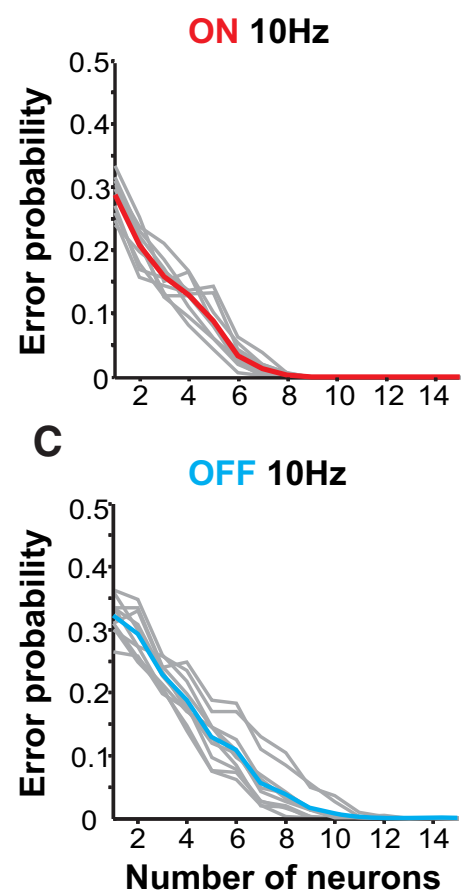

B
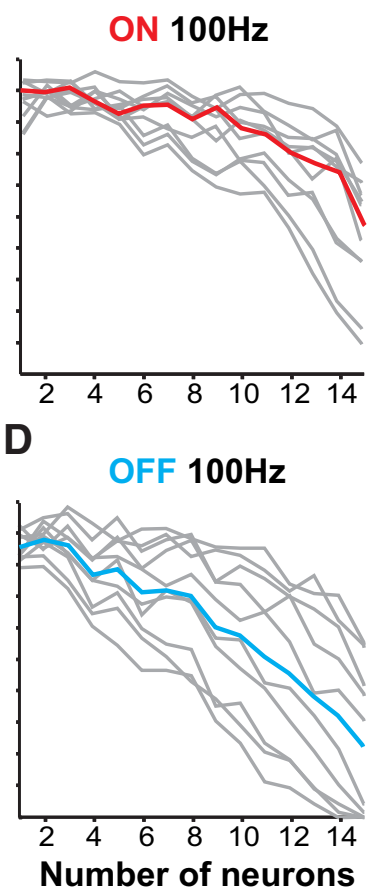

Figure 2. Detection efficiency depends on beat frequency. $\boldsymbol{A}$, Detection of chirps on $10 \mathrm{~Hz}$ beat by ON cells as a factor of neurons included in the analysis $(n=17)$. Error probability is the probability of an ideal observer to correctly assign a spike train as a chirp or beat response. Detection error levels for individual chirp identities are shown in gray. Red line indicates mean detection error for all chirps. ON cells can reliably detect the occurrence of all chirps. B, ON-cell performance is worse on 100 $\mathrm{Hz}$ beats. $\boldsymbol{C}, \boldsymbol{D}$, Mean OFF-cell performance (cyan, $n=16$ ) is also more efficient on a $10 \mathrm{~Hz}$ beat.

the terminology used in $A$. lepthorhynchus to describe the two ends of the spectrum of chirp properties (small, $<150$ $\mathrm{Hz}$ increase; big, $>150 \mathrm{~Hz}$ ). Figure 3 shows discrimination ability for chirps grouped by size (Table 1; Small chirps: 5 , 6, 7, 8; Big chirps: 1, 2, 3, 4, 9, 10) for both ON and OFF cells on high and low beat frequencies.

Chirps of all types are more easily discriminable when presented on $10 \mathrm{~Hz}$ beats (Fig. $3 A$ ) rather than on $100 \mathrm{~Hz}$ beats (Fig. 3B). Performance for chirps on high-frequency beats was variable: accurate discrimination was achieved only for chirps with large differences in properties (Extended Data Fig. 3-1), and both ON and OFF cell responses allow qualitatively similar discrimination accuracy. These results are unexpected, considering that the same analysis in $A$. leptorhynchus shows that chirps on high-frequency beats can be discriminated well using as few as six neurons, and conversely exhibit poorer discrimination on low frequencies (Marsat and Maler, 2010) where A. albifrons performs best. Furthermore, we do not observe an asymmetry in coding accuracy between ON and OFF cells, as observed in $A$. leptorhynchus.

When looking at which chirps are well discriminated, chirp duration and the steepness of the frequency rise seem to be the most discriminable features, while total frequency increase and the number of peaks are less 
A
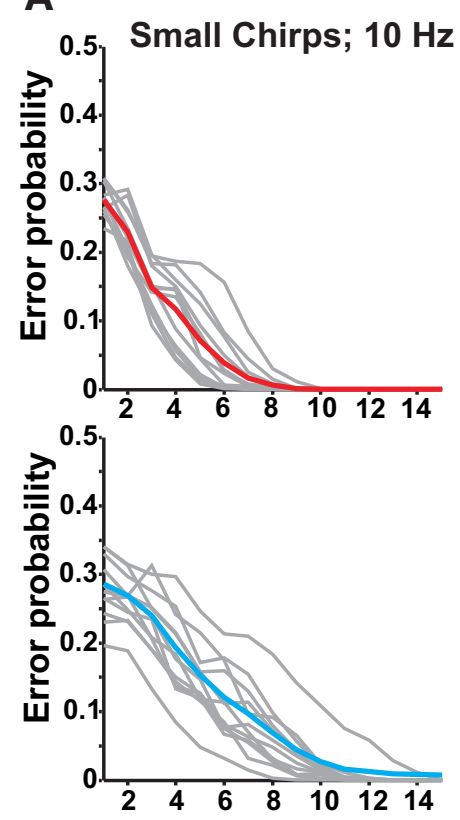

B
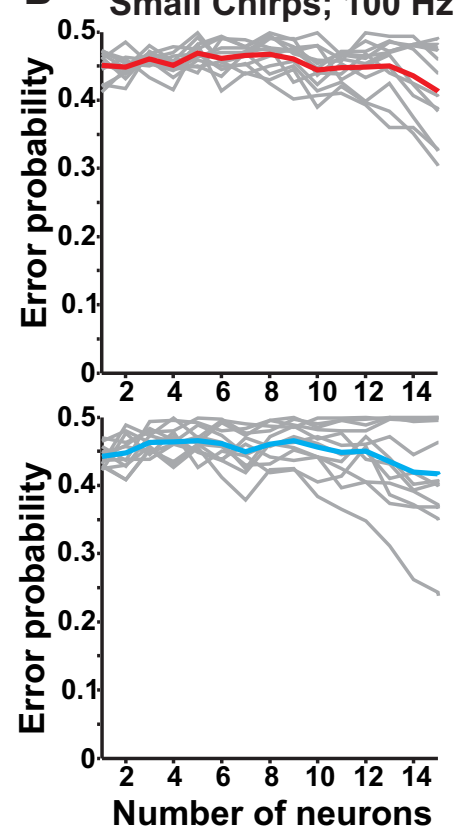

Big Chirps; $10 \mathrm{~Hz}$
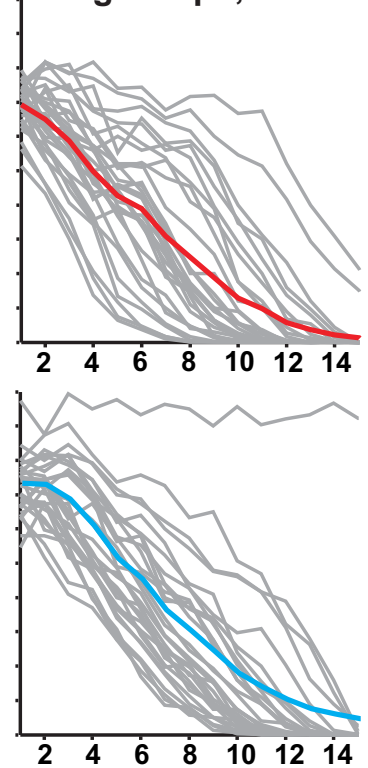

Big Chirps; $100 \mathrm{~Hz}$
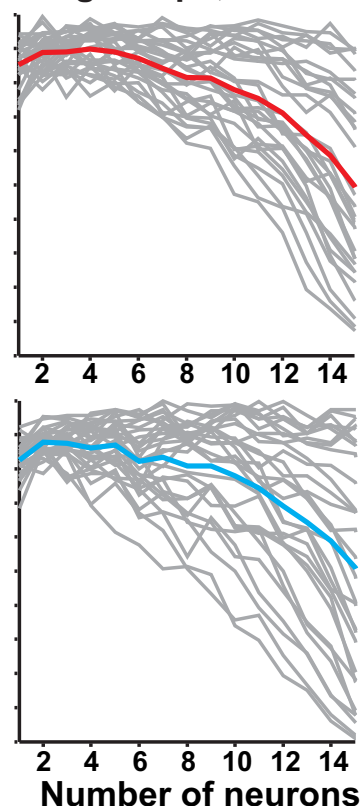

Figure 3. Discrimination of chirps on high-frequency beats is poor. A, Discrimination for small chirps (chirps 5, 6, 7, 8, right) and big chirps (chirps 1, 2, 3, 4, 9, 10, left) on $10 \mathrm{~Hz}$ beat. Mean ON-cell chirp discrimination shown in red, OFF cells are in cyan, and discrimination for individual chirp pairs are shown in gray. $\boldsymbol{B}$, Discrimination of small chirps on a $100 \mathrm{~Hz}$ beat is relatively inefficient for both ON (red) and OFF cells (cyan). Discrimination of big chirps varies with chirp identity, but is still poor. For chirp-by-chirp discrimination comparisons, see Extended Data Figure 3-1.

influential on coding (Extended Data Fig. 3-1). However, discriminating the most easily separated chirps is prone to error and requires a larger pool of neurons on highfrequency beats. These data indicate that while qualitative

observations would suggest that chirp coding is similar between $A$. albifrons and $A$. leptorhynchus, there are quantitative differences in the information content about chirps within the electrosensory system. To determine which aspects of the neural responses could be responsible for changes in chirp coding, we characterized a wide variety of response properties using an assortment of stimulation protocols.

\section{Frequency tuning and neural coding}

Encoding AMs in stimuli is crucial for the perception of social signals. As shown above, A. albifrons neurons respond variably to chirps, which consist of both highfrequency $\mathrm{AM}$ signals and the low-frequency changes in the contrast of the AM (i.e., the envelope). We asked whether the accuracy of detection and the discrimination of chirps by $A$. albifrons ELL neurons reflect speciesspecific frequency-tuning properties.

Communication signals cause spatially diffuse stimulation compared with the localized electric fields of prey items. We simulate this difference by altering the geometry of our stimulating dipoles. Using a large dipole (see Materials and Methods) that simulates diffuse communication signals drives both the feedforward responses from electroreceptor to pyramidal neuron and the feedback pathways driven by broad receptive fields. To separate the effects of feedback from the tuning of the feedforward circuit, we also stimulated only the receptive field of each cell with a small local dipole simulating localized objects or prey (Bastian, 1986; Chacron et al., 2005).

In response to simple sine waves, $A$. albifrons exhibits the highest degree of coding fidelity at low frequencies. The firing rate for each sine cycle peaks at $5 \mathrm{~Hz}$ for all cell types and stimulation configurations (Fig. 4A). Maximum phase locking also peaks at low frequencies (Fig. 4B). These values closely match previously reported values (Martinez et al., 2016). Temporal coding accuracy is conveniently quantified using random noise stimuli and information theory (Borst and Theunissen, 1999). Lowerbound coherence reflects the amount of information encoded linearly, whereas upper-bound coherence takes into account both linear and nonlinear aspects of the response (Fig. 4C). Both ON- and OFF-cell coherence curves peak at similar frequencies in the $5-20 \mathrm{~Hz}$ range (Wilcoxon rank-sum test, $p=0.19$ ). However, OFF cells are clearly low pass and exhibit a sharp decline in coherence to higher frequencies, while ON cells exhibit a slightly broader lower-bound coherence (Fig. 5; see accompanying text for more details).

The movement of fish as well as the amplitude changes caused by chirps create low-frequency changes in AM contrast, or envelopes (Stamper et al., 2013). During chirps, the AM will be high frequency (tens to hundreds of hertz above the beat frequency), whereas the envelope of the chirp is low frequency (e.g., a $100 \mathrm{~ms}$ chirp will lead to an $\sim 10 \mathrm{~Hz}$ envelope). Therefore, the coding of the lowfrequency content of chirps must be investigated using envelope stimuli. We characterized envelope coding using stimuli consisting of RAMs with AM frequencies between 
A
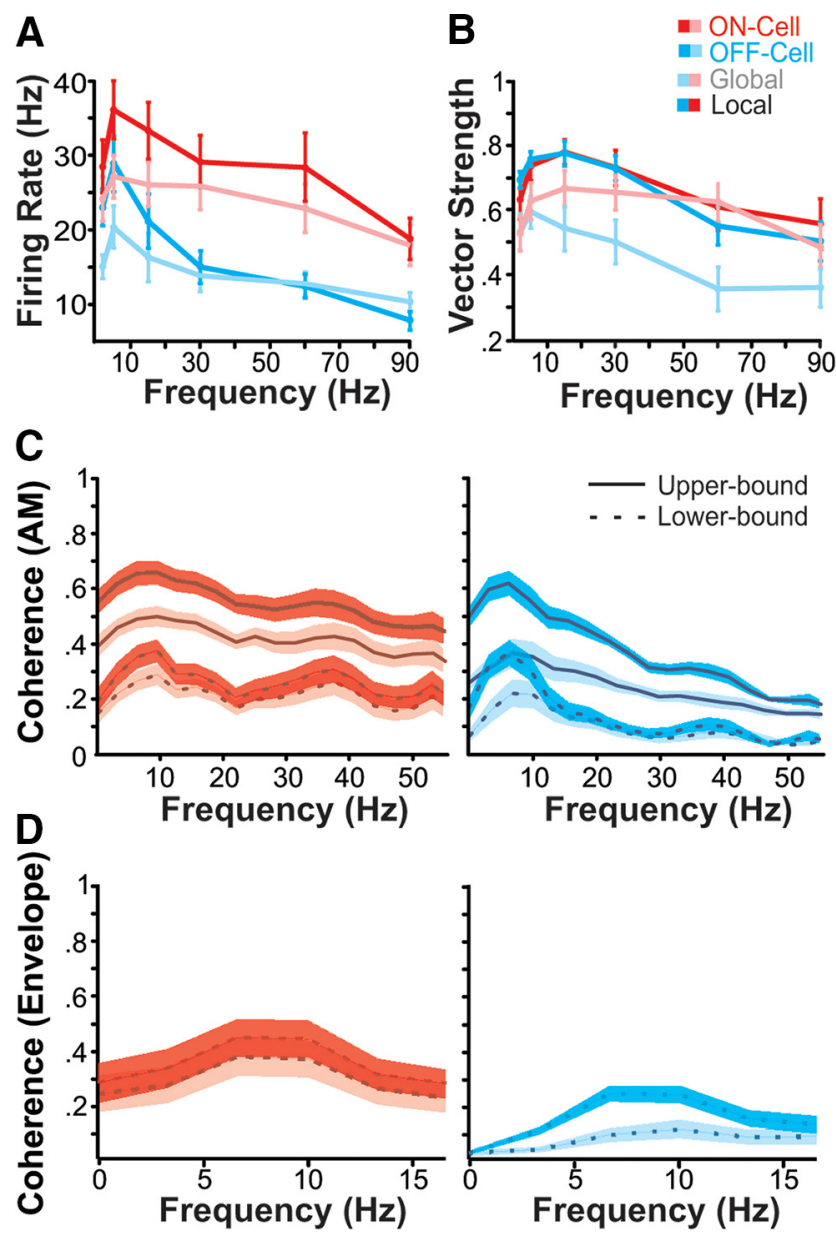

Figure 4. Temporal coding properties of pyramidal cells. A, Mean firing in response to SAM stimuli. Mean firing rate $( \pm S E)$ for both ON (red, $n=19$ ) and OFF (cyan, $n=15$ ) cells in both stimulation configurations (global: pink and pale blue; local: red and cyan) peaks at $5 \mathrm{~Hz}$. B. Phase locking to AM sinusoids is also best at low frequencies. Maximum phase locking is seen at $15 \mathrm{~Hz}$, with the exception of locally stimulated OFF cells, which peak at $5 \mathrm{~Hz}$. C, Mean coherence to noise stimulation is also low pass. ON-cell coherence is shown in red, OFF-cell in cyan. The upper-bound coherence measure (solid line) is based on the response-response correlations between multiple presentations of the stimulus, while the lower bound (dashed line) is based on stimulus-response correlations. Shaded areas indicate SE; darker shading indicates local stimulation. Mean global lowerbound maximums: ON cells, $23.1 \mathrm{~Hz}$ ( $\pm 0.86 \mathrm{SE})$; OFF cells, $11.6 \mathrm{~Hz}( \pm 0.75 \mathrm{SE}$; Wilcoxon rank-sum test, $p=0.04)$. $\boldsymbol{D}$, Coding of low-frequency envelopes is poor. Mean ( $\pm S E$ ) lower-bound coherence between responses and the lowfrequency $(0-20 \mathrm{~Hz})$ envelope of a bandpass RAM stimulus $(40-60 \mathrm{~Hz})$ are displayed for both local and global stimulus configurations. Both ON and OFF cells exhibit peak envelope tuning at $10.10 \mathrm{~Hz}( \pm 1.52 \mathrm{SE}$; Wilcoxon rank-sum test, $p=$ $0.21)$. OFF cells have noticeably lower coherence to low envelopes than $\mathrm{ON}$ cells.

40 and $60 \mathrm{~Hz}$, containing envelope frequencies of $0-20$ $\mathrm{Hz}$ (Middleton et al., 2006). Although pyramidal cells do encode the envelope of these stimuli (Fig. 4D), the coding accuracy is low compared with the envelope coding observed in A. leptorhynchus (Chacron, 2006; Middleton et al., 2006; Extended Data Fig. 5-1). OFF-cells are particularly relevant for chirp discrimination. This is because chirps consist of decreases in envelope amplitude, which are best encoded by OFF cells (Marsat and Maler, 2010). The observed poor coherence to envelope stimuli, particularly for OFF cells in receiving global stimulation, may in part cause the poor chirp discrimination that we observe.

\section{Burst firing and chirp detection}

As described in the study by Marsat et al. (2009), burst firing serves an important role in A. leptorhynchus for the efficient detection of extremely short chirps. As qualitatively shown above in Figure 1, no conspecific chirps reliably produced a burst response in $A$. albifrons. This implies that $A$. albifrons may not be using this dedicated code for the detection of very brief chirp signals. In $A$. leptorhynchus, the coding of small chirps is directly related to beat frequency. On low frequencies, small chirps are shorter than the period of one beat cycle. As beat frequency increases and the period becomes shorter, small chirps begin to last longer than one cycle. This change in duration relative to beat period mediates a change in how small chirps are coded (Walz et al., 2014). In $A$. albifrons, all chirps are much longer than $A$. leptorhynchus small chirps, spanning more than one beat cycle even on low frequencies, which may eliminate the need for a feature detection code specifically for extremely short chirps. Thus, the observed lack of bursting may result from the change either in signal structure (duration relative to beat cycle) or from underlying changes to the physiology and bursting capability of LS neurons.

To determine whether this change in signal coding is a result of signal structure or underlying physiology, we stimulated $A$. albifrons fish with $A$. leptorhynchus chirps known to elicit a burst response in $73 \%$ of $A$. leptorhynchus LS ON cells (Marsat et al., 2009). We determined the burst threshold ISI for each neuron by plotting ISI distribution of chirp and beat responses and manually selecting a threshold that best distinguished between the two (Martinez-Conde et al., 2002) in order to maximize our measure of chirp-specific bursting. The mean \pm SE burst threshold was $8.0 \pm 0.7 \mathrm{~ms}$, which is comparable to the burst thresholds determined for our RAM analyses and in other reports (Chacron and Bastian, 2008; Marsat et al., 2009; Avila-Akerberg et al., 2010).The majority of neurons increased firing rate only slightly more in response to $A$. leptorhynchus chirps than to the beat. There was, however, a small percentage $(\sim 20 \%)$ of ON cells that did reliably burst more in response to $A$. leptorhynchus small chirps than to the beat (Fig. 5A,C). Most importantly, even the bursty neurons did not respond to the shortest $A$. albifrons chirps with bursts (Fig. $5 B, D$ ). Unlike A. leptorhynchus small chirps, which may cause either amplitude increases or decreases, depending on chirp time in relation to beat phase, $A$. albifrons chirps are all long enough to span more than one beat cycle. This chirp length in relation to the beat period means that the AM waveform caused by chirps is less variable and that the response they elicit is largely independent of the phase at which chirps start. This may mean that having a chirp-invariant 
A

\section{A. leptorhynchus Chirp}

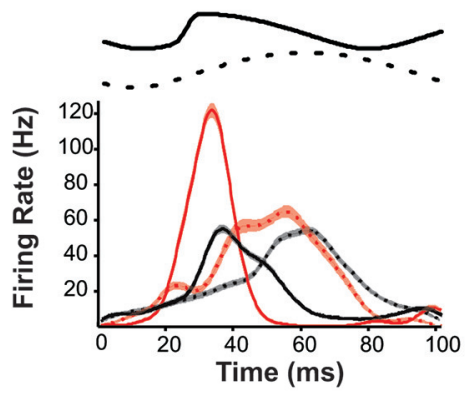

B

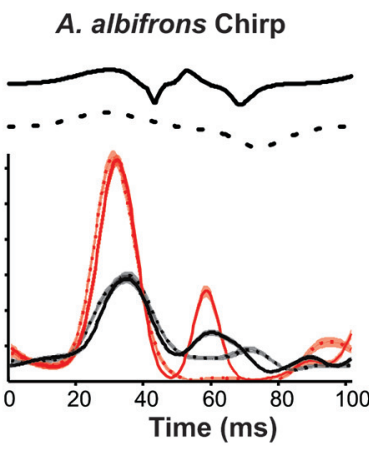

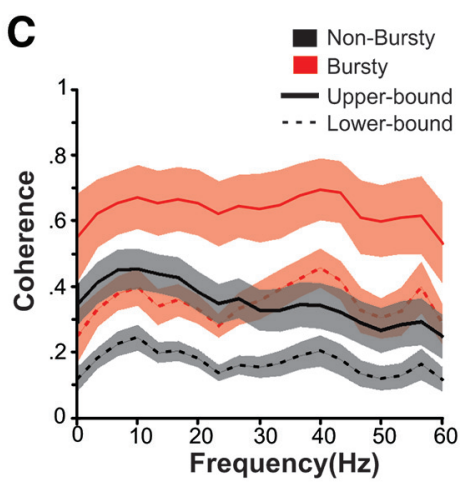

D

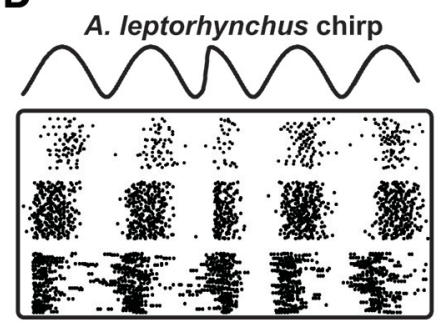

A. leptorhynchus chirp

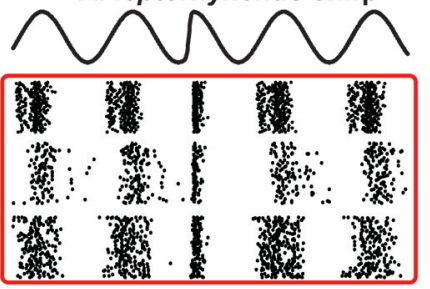

Figure 5. Coding of chirps by a small population of higher-pass neurons. $\boldsymbol{A}$, The mean instantaneous firing rates of ON cells over the time course of chirp and beat stimuli. The difference in peak firing rate(FR) characterize a small population $(n=5$, red) that bursts in response to $A$. leptorhynchus small chirps (beat responses: dashed lines; peak FR, $77.67 \pm 7.11 \mathrm{~Hz}$; chirp responses: solid lines; peak FR, $159.57 \pm 8.68 \mathrm{~Hz}$; Wilcoxon rank-sum test $(\rho<0.001)$. The majority of the ON-cell population $(n=16$, black) only showed a modest increase in firing (beat response: peak FR, $62.68 \pm 1.27 \mathrm{~Hz}$; chirp response: peak FR, $66.56 \pm 4.21 \mathrm{~Hz}$; Wilcoxon rank-sum test, $p=0.002$ ). Shaded area represents SE. $\boldsymbol{B}$, Even the bursty population fires less in response to $A$. albifrons smallest chirps compared with the beat (beat response: peak FR, $155.45 \pm 8.82 \mathrm{~Hz}$; chirp response: peak FR, $117.75 \pm 9.40 \mathrm{~Hz}$; Wilcoxon rank-sum test, $p=0.04)$. $\boldsymbol{C}$, Mean ( $\pm \mathrm{SE}$ ) upper-bound and lower-bound coherences for the bursting (red) and nonbursting (black) populations. $\boldsymbol{D}$, Example raster plots of chirp responses used for $\boldsymbol{A}$ and $\boldsymbol{B}$. The nonbursty population (black box) responds similarly to both $\boldsymbol{A}$. leptorhynchus chirp and beat. The bursty population (red box) bursts to $A$. leptorhynchus chirps, but not $A$. albifrons chirps. For comparable data obtained from A. leptorhynchus, see Extended Data Figure 5-1.

coding mechanism, such as bursting, is not necessary in this species.

The low proportion of neurons that burst in response to A. leptorhynchus chirps demonstrates that there are physiologic differences between these two species in regard to the burst coding of communication signals. We examined the differences between bursty and nonbursty neurons in more detail by analyzing separately their responses to RAM stimuli. The subset of bursty neurons have broader AM tuning (Fig. 5C), as tested by comparing the ratio of coherences summed over the $0-30$ versus $30-60 \mathrm{~Hz}$ (bursty cells mean ratio $\pm \mathrm{SD}, 0.91 \pm 0.12$; nonbursty cells mean ratio, $2.35 \pm 1.72 ; t$ test not assuming equal variance, $p=0.0047$; similar results were found using the upper-bound coherence). While most ON cells exhibited peak coherence at frequencies between 10 and $20 \mathrm{~Hz}$ (nonbursty cells mean peak frequency \pm SD, 19.6 \pm 18 ), these cells had significantly higher peak frequencies as high as $40-50 \mathrm{~Hz}$ (mean $\pm \mathrm{SD}, 40 \pm 13.9 \mathrm{~Hz} ; t$ test, $p=0.023$ ) and exhibited high coherence across the range of stimulus frequencies. The remainder of the ON cells displayed the low-pass tuning described in the study by Martinez et al. (2016). These data show that while a subset of ELL neurons is capable of bursting in response to short chirp stimuli, they make up a small percentage of the overall ELL population and bursting does not seem to play a role in conspecific chirp coding.

\section{Feature detection}

Burst firing may not be a significant aspect of communication coding in $A$. albifrons, but other uses for burst coding may be conserved. During spontaneous activity, we observed a baseline firing rate of $13.54 \mathrm{~Hz}( \pm 1.37 \mathrm{SE}$ ) with $17.01 \%( \pm 2.55 \mathrm{SE})$ of spikes in bursts. Thus, the pyramidal neurons of $A$. albifrons are physiologically capable of bursting. In $A$. leptorhynchus, burst firing can reliably signal the presence of spatially localized prey-like stimuli (Gabbiani et al., 1996; Oswald et al., 2004). We examined bursting in response to local RAM stimulation (Fig. $6 A$ ) to determine whether bursting could serve similar prey detection functions in $A$. albifrons. The ISI histogram of the responses clearly showed that the neurons burst to these stimuli (Fig. 6B). The proportion of spikes occurring in bursts was as high as $63.07 \%$ ( $\pm 5.64 \mathrm{SE}$ ) for stimuli in the local configuration (Fig. 6C). The average stimulus waveform triggering burst versus single spikes follows the pattern observed in other species: slower AM for bursts than for single spikes (Fig. 6D). However, in response to RAM stimuli, unlike $A$. leptorhynchus there was not a large difference in coding error between bursting and tonic spiking (ANOVA, $p=0.10$; Fig. $6 E$ ). These data suggest that while bursts encode low-frequency stimuli in the LS of $A$. albifrons, they do not appear to implement a burstbased feature detection code for either prey-like stimuli or chirps. 

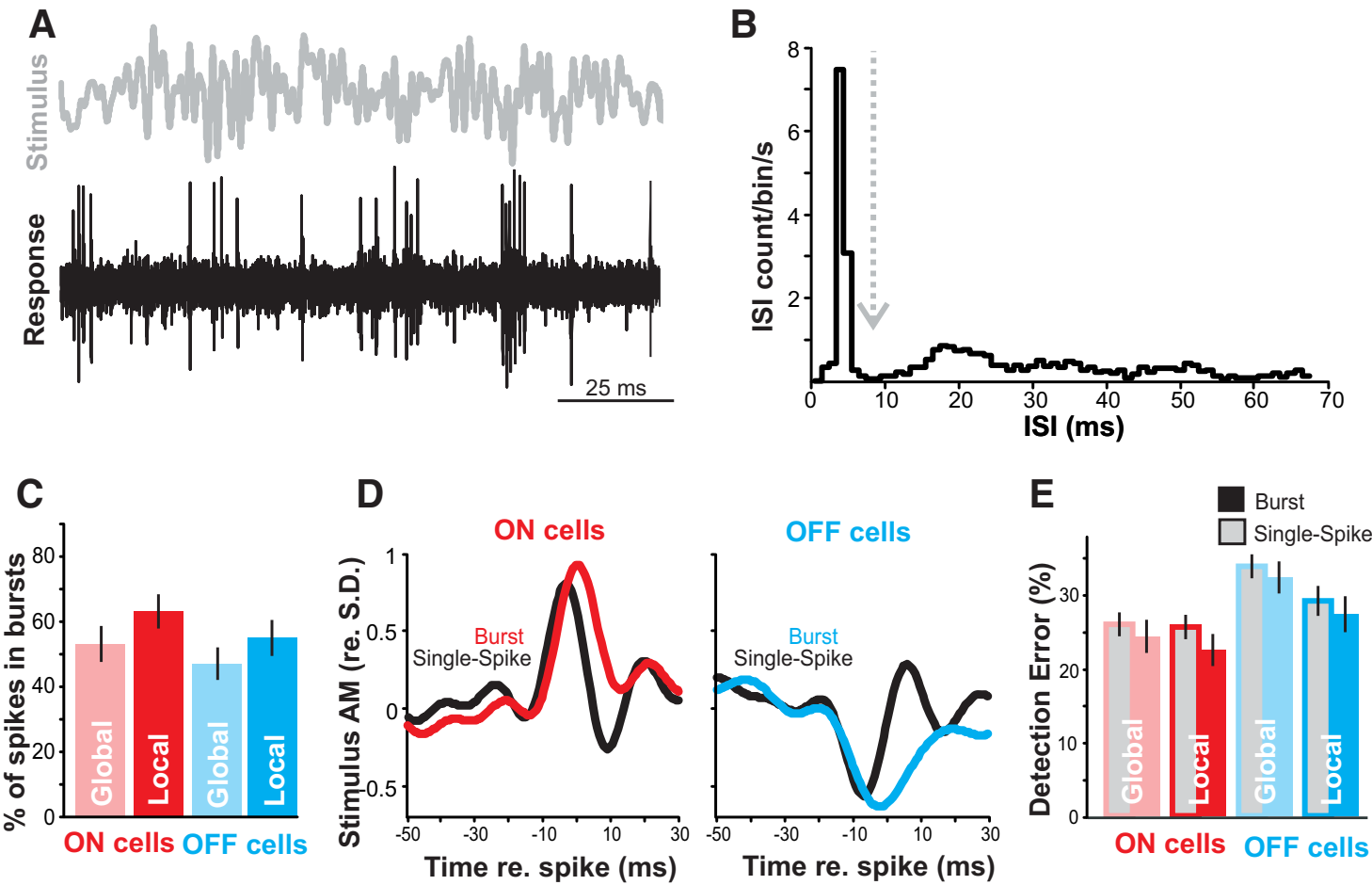

Figure 6. Coding of AM by bursts. $\boldsymbol{A}$, Sample of noise stimulus $(0-60 \mathrm{~Hz}$, gray) and representative spike train (black) from an OFF cell during local stimulation. $\boldsymbol{B}$, Example of ISI distribution used to determine burst threshold (dashed line). $\boldsymbol{C}$, ON cells (red) burst more than OFF cells (blue; ANOVA, $p=0.02$ ), and local stimulation produced more bursting than global stimulation (ANOVA, $p=0.01$ ). Error bars show the SE. $\boldsymbol{D}$, Mean burst-triggered averages (red/blue) and single spike-triggered averages (black) from ON and OFF cells show that bursts are triggered by wider (lower-frequency) stimulus features than single spikes. $\boldsymbol{E}$, Feature detection performance for burst and isolated spikes. In both ON- and OFF-cell bursts (blue/red-filled bars) tend to have lower error rates (percentage of events signaling false positives or false negatives) in detecting optimal stimulus features than single spikes (gray-filled bars), but this trend is not significant (ANOVA, $p=0.10$ ).

\section{Chirp production and behavior}

Our physiology data suggest that $A$. albifrons is able to detect and discriminate chirps on low-frequency beats much more sensitively than on high-frequency beats. To test this hypothesis, we recorded electrical behavior from pairs of freely swimming and interacting fish. Chirping behavior did occur but differed from that of $A$. leptorhynchus in a number of ways. Primarily, overall rates of chirping are dramatically lower than the numbers reported from similar studies in A. leptorhynchus (Zupanc et al., 2006; Hupé and Lewis, 2008). Over 28 trials, we recorded a sum of 133 chirps, with a mean of $4.75( \pm 0.61 \mathrm{SE})$ chirps per 5 min trial, whereas in a similar context male $A$. leptorhynchus produced $\sim 125$ chirps per 5 min trial (Hupé and Lewis, 2008). The maximum number of chirps observed in one trial was 13. Due to the small number of observed chirps, we did not separate them into multiple categories for analysis, although chirps of varying frequency increases and duration occurred. Chirping frequency is correlated with the difference in EODfs of interacting fish. Smaller differences in EODf correspond to higher numbers of chirps (Fig. 7A). However, under our conditions chirping does not appear to be sexually dimorphic. Animals used in behavior experiments were not killed to determine sex, but we grouped them by EODf into high-frequency $(>1100 \mathrm{~Hz})$ and low-frequency $(<1100 \mathrm{~Hz})$ groups, which can correspond to females and males, respectively, in many populations of $A$. albifrons (Zakon and Dunlap, 1999). We observed no differences in chirp production between high- or low-frequency groups (Fig. 7B). Further, chirp rate does not vary by pairing type, or by relative EODf of individuals within each pair under our conditions (Fig. 7B). These results confirm previous findings that chirp frequency in this species is not sexually dimorphic (Dunlap et al., 1998).

Chirp timing plays an important role in mediating $A$. leptorhynchus interactions as they frequently exchange chirps in an echo response pattern (Zupanc et al., 2006; Hupé and Lewis, 2008; Henninger et al., 2018), a behavior where one fish responds to the chirp of another fish by chirping back within $<1 \mathrm{~s}$. To determine whether chirp timing plays a similar role in $A$. albifrons interactions, we analyzed interchirp intervals for all trials in which both fish chirped. The resulting distribution of all chirp latencies between fish indicates interchirp latencies much longer than are typical of $A$. leptorhynchus echo responses (Fig. $7 C)$. Of all recorded chirps, only two occurred within $2.5 \mathrm{~s}$ of each other, making an echo response unlikely to be an important feature of $A$. albifrons interactions. Furthermore, interchirp intervals for a given fish (Fig. 7D) follow a Poisson distribution, indicating that chirp production is not produced in stereotyped sequences. The lack of echoing, the long interchirp intervals, and the low rate of chirping in general suggest that $A$. albifrons does not rely 


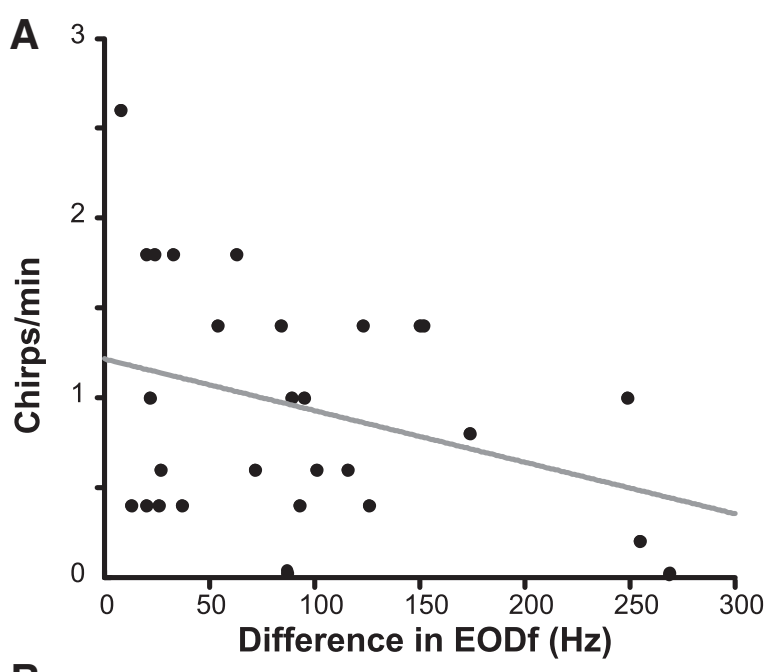

B
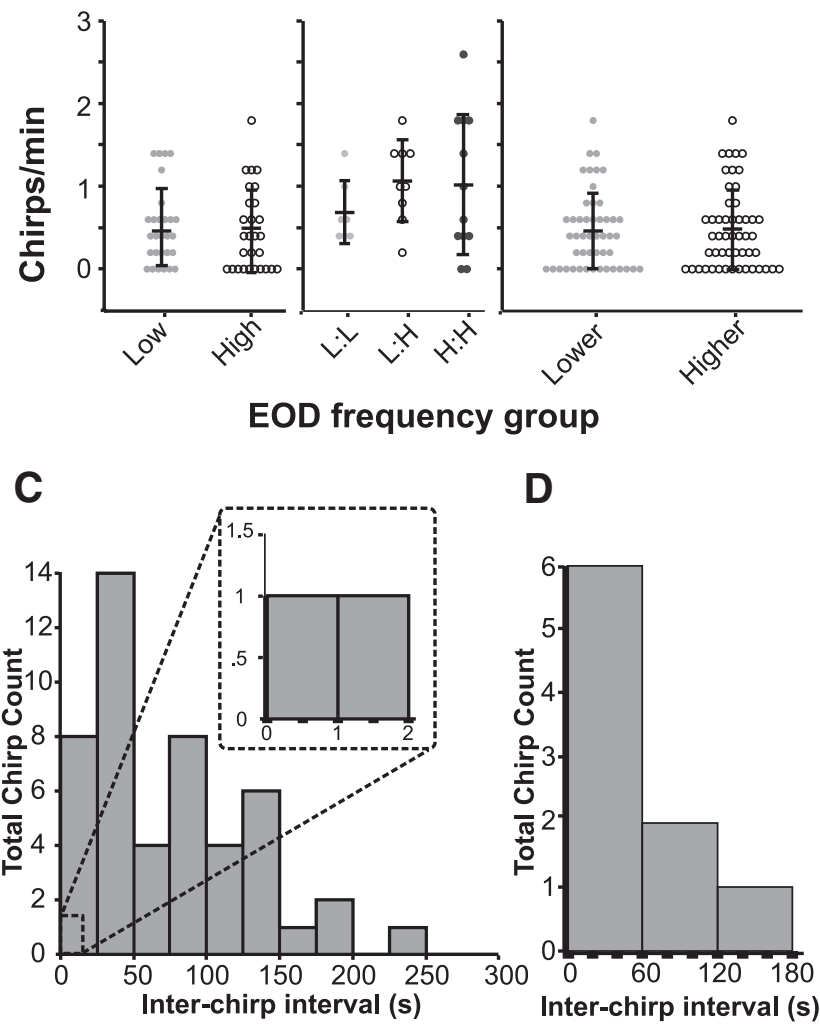

Figure 7. Chirping behavior in freely swimming pairs $(n=28)$. $\boldsymbol{A}$, The number of chirps produced during interaction is correlated with difference in EODf $\left(r^{2}=0.1093\right)$. $\boldsymbol{B}$, Chirping does not differ by absolute EODf, EODf pair type, or relative EODf. The mean chirp rate for low-frequency fish $(<1100 \mathrm{~Hz})$ and high-frequency fish $(>1100)$ was similar (Student's $t$ test, $p=0.86$ ). Mean chirps per trial based on the EODf of pairing [Low:Low (L:L); Low:High $(\mathrm{L}: \mathrm{H})$; High:High $(\mathrm{H}: \mathrm{H})$; ANOVA, $p=0.47]$ and by the relative frequency of individuals within the pairing (Student's $t$ test, $p=$ $0.55)$ were all extremely low and similar in all groupings. Error bars indicate SD. C, Interchirp intervals between pairs binned by time fall show no echoing chirp exchanges. Inset, Enlarged section shows that very few chirps occur within $2 \mathrm{~s}$ of each other. $\boldsymbol{D}$, Interchirp interval distribution for individual fish follow a Poisson distribution $\left(R^{2}=0.9\right)$. on the detailed decoding of individual chirp characteristics, as is the case in A. leptorhynchus.

\section{Discussion}

We found that neurons in the primary sensory area of $A$. albifrons are able to detect chirp occurrence and do encode chirp identities on low-frequency beats. However, they discriminate between chirps less accurately when chirps are presented on high-frequency beats. Rather than producing discrete burst responses for small chirps and tonic responses for big chirps, all chirps in this species are encoded via graded and heterogeneous increase or decreases in firing rate. We found several adaptations in the basic response properties that may explain the neural responses to conspecific chirps. These adaptations, frequency tuning in particular, may facilitate encoding long chirps in $A$. albifrons as well as enhancing chirp coding in the behavioral context in which chirps are used most often. Therefore, we also asked whether chirping behavior could explain differences in chirp coding efficiency for high- and low-frequency beats. We found that the pattern of chirp use during common social interactions is very different from those of closely related species: $A$. albifrons fish produce very few chirps and do not respond to each other with echo chirps. Infrequent chirping is particularly pronounced in the context of high-frequency beats, suggesting that certain types of interactions are not mediated by chirps. We suggest that the observed differences in chirp coding accuracy on high-frequency beats may reflect a relaxation in the demands for exchanging information via chirps in this context.

\section{Low-frequency tuning could be adaptive for chirp coding}

Our results confirm that pyramidal cells in the LS of $A$. albifrons are low pass. This finding parallels the lowfrequency envelopes produced by long $A$. albifrons chirps. However, the impact of this AM tuning on chirp coding depends on the way AMs and envelopes are processed in this system. Whereas the envelope of chirps is low frequency, the AM is high frequency. Therefore, low-frequency tuning to AM is not automatically helpful. We found that the coding of low-frequency envelopes was relatively poor, particularly in OFF cells, which, in $A$. leptorhynchus, are best at coding chirp envelopes. However, if envelope response is synthesized before the pyramidal cells (e.g., at the electroreceptors, as could be the case in A. leptorhynchus; Savard et al., 2011), lowfrequency tuning to AM could benefit chirp coding since the input to the pyramidal cells would contain low frequencies (Metzen et al., 2018). Nevertheless, we show that the neural response does not accurately reflect the chirp envelope and that envelope coding of decreases in the amplitude of RAM envelope stimuli is poor. Our data replicate the findings of Martinez et al. (2016) that also show poor coding of envelope stimuli, with coherence values of $\sim 0.1$. It is possible that without low-frequency tuning, chirp and envelope coding would be even worse. Thus, low-frequency tuning may compensate for other properties that hinder envelope coding (e.g., the amount of rectification performed by the receptors; Savard et al., 2011). 


\section{The role of burst coding}

Bursts also often play an important role in coding specific features of communication signals (Creutzig et al., 2009; Fujimoto et al., 2011) as is the case for A. leptorhynchus, where bursting enhances the detectability of small chirps on low-frequency beats (Marsat et al., 2009). A. leptorhynchus small chirps are unusual in that, for low-frequency beats, they span less than a full cycle of the beat. Consequently, the same chirp can be perceived as a sharp decrease in amplitude, a sharp increase, or a mix of the two, depending on the phase of the beat at which the chirp starts (Benda et al., 2005). It may be because of this aspect of chirp structure and its shortness that the sensory system of $A$. leptorhynchus uses bursts to enhance detectability rather than optimizing the coding of the properties of these chirps. A. albifrons is not subject to the same constraints. The perceived AM of a chirp is largely independent of beat phase since chirps span several cycles of the beat and their long duration might make them more conspicuous (Petzold et al., 2016). Using noise stimuli, we showed that burst coding in the LS is different in $A$. albifrons. Since bursts are not used to encode chirps, and because LS primarily focuses on processing communication signals, it is possible that these neurons do not need to use burst as a feature detection signaling mechanism, as in other species (Gabbiani et al., 1996; Oswald et al., 2004).

\section{Chirp coding and behavior}

Our chirp response analysis shows that all chirps are coded with graded increases and decreases in firing rate containing some information about chirp properties but, for high-frequency beats, coding accuracy is inefficient at supporting both chirp detection and discrimination. Our behavioral data might shed some light on this apparent inefficiency at coding chirps across all social contexts. We show that, in our conditions, $A$. albifrons chirp relatively infrequently to mediate dyad interactions. Furthermore, we show that there is a tendency to chirp less when the beat frequency is higher. This is supported by previous findings showing that chirp production is more frequent in interactions involving low-frequency beats (Kolodziejski et al., 2007), reiterating the relevance of chirp coding on low-frequency beats rather than high-frequency interactions.

\section{Comparison to A. leptorhynchus and other electric fish}

Our physiology and behavioral findings are markedly different than those observed in the closely related $A$. leptorhynchus. Primarily, our data show $A$. albifrons fish exhibit poor chirp coding on high-beat frequencies, and efficient coding on low-beat frequencies. This is directly in contrast with $A$. leptorhynchus fish, which encode chirps on high-frequency stimuli well and exhibit poorer coding of chirp identity on low-frequency beats (Marsat and Maler, 2010; Allen and Marsat, 2018). Furthermore, we find that $A$. albifrons fish do not rely on the same coding strategy as used in A. leptorhynchus since feature detection through bursts is not involved in chirp coding. Our data clearly demonstrate that, despite being so closely related (Smith et al., 2016), the sensory coding properties of the neurons encoding communication signals have diverged between $A$. leptorhynchus and $A$. albifrons. Chirps have been suggested to be short in the ancestors basal to the two species (Smith et al., 2016) and longer chirps to be a development of the A. albifrons branch. Therefore, it is possible that the differences in frequency tuning we observed in $A$. albifrons may be adaptive for coding these long chirps.

Our behavioral results suggest that $A$. albifrons fish also use chirp exchanges very differently than $A$. leptorhynchus. Unlike the complex chirp interactions observed in A. leptorhynchus, where chirps are omnipresent and play a central role in various types of interactions (Hagedorn and Heiligenberg, 1985; Hupé, 2012; Henninger et al., 2018), A. albifrons fish chirp infrequently and generally only in low-frequency difference interactions (Kolodziejski et al., 2007). While $A$. leptorhynchus fish have dedicated uses for different chirp types (Henninger et al., 2018), A. albifrons fish use all types of chirps interchangeably for low-beat frequency interactions and may not possess distinct chirp categories (Dunlap et al., 1998; Kolodziejski et al., 2007; Turner et al., 2007). The link between chirp properties and behavior in $A$. albifrons is also less clear than that in A. leptorhynchus (Dunlap and Larkins-Ford, 2003). Therefore, we suggest that rather than having distinct coding strategies for different chirp types, they have adopted context-specific coding to better encode chirps on low-frequency beats where chirps are most likely to be produced.

Chirping behavior has been investigated across many species of wave-type gymnotiforms (Hagedorn and Heiligenberg, 1985; Turner et al., 2007; Petzold et al., 2016, 2018). Details of chirp encoding, however, have only been investigated in one other species, Eigenmannia viscerens (Metzner and Heiligenberg, 1991; Metzner and Viete, 1996; Stöckl et al., 2014). In this species, similarly to $A$. leptorhynchus, different chirp types are used to mediate agonistic and courtship encounters (Hagedorn and Heiligenberg, 1985). In this species, unlike the apteronotids, chirp coding appears to be unaffected by beat frequency, at least at the level of electroreceptors (Stöckl et al., 2014). However, the coding strategy observed in the ELL pyramidal cells is that the duration of the chirp is the primary feature encoded. This feature is coded via brief excitation, then inhibition of both ON and OFF cells (dependent on the sign of the DC component of the chirp) that signals the duration of the chirp (Metzner and Heiligenberg, 1991). This strategy is similar to the coding observed in $A$. albifrons in low-frequency interactions, suggesting that perhaps $A$. leptorhynchus represents the outlier group, and burst detection of chirps is a specialization for their unique small chirps.

\section{Conditions affecting chirp coding}

Our study did not test for the effects of neuromodulation on neural tuning and chirp coding. Neuromodulation can change cell response properties for different behavioral states (Harris-Warrick and Marder, 1991). Previous 
work in A. leptorhynchus shows that serotonin enhances pyramidal cell excitability and responsiveness to small chirps on low-frequency beats (Deemyad et al., 2013). While we worked on adult animals, we did not determine sex or breeding status, both of which have large effects of the frequency and quality of chirps produced (Smith, 2013). It is likely that the effects of neuromodulation due to behavioral state could affect the reception and encoding of these chirps as well, altering the sensitivity to chirps and possibly even the coding accuracy in response to behavioral need. This may particularly influence the coding of chirps on high-frequency beats, which we observed was surprisingly poor. This kind of interaction is more likely to occur in breeding contexts, so it is possible that animals in breeding condition could be better able to detect and discriminate these signals than the results we show here. We also did not investigate other environmental factors that could influence the sensory specializations of both species, such as microhabitats, prey capture, and general sociality, all of which could drive some of the adaptations seen.

Our analysis technique is widely used to quantify the encoding performance of sensory neurons (Neuhofer and Ronacher, 2012; Itatani and Klump, 2014; Mouterde et al., 2017), and is biologically realistic (Larson et al., 2009). Nevertheless, other analysis techniques could be devised to improve the discrimination accuracy estimate. Weighting the contribution of the different neurons or weighting them differently across time could be envisioned (Larson et al., 2010). Keeping the different neurons as separate dimensions in the analysis (Houghton and Sen, 2008) or combining ON- and OFF-cell responses (AumentadoArmstrong et al., 2015) could also improve discrimination, as could accounting for neural correlation in response variability (Hofmann and Chacron, 2017). Finally, various more complex decoding methods (e.g., multilayered neural net or principal component analysis (PCA)-type dimensionality reduction) could be used for analysis. Nevertheless, we do not expect the neural mechanism to differ widely across apteronotid species, so our analysis provides a meaningful comparison. The strength of our measure is that it is conservative: it gives a clear account of the information available in ELL pyramidal cells with few assumptions about what the downstream networks use for decoding.

Trade-offs between specialization and generalization

Classical neuroethology dictates that the mode of signal production and mode of signal reception must evolve in synchrony so that senders and receivers never lose the ability to exchange information (Bradbury and Vehrencamp, 2011). There are many examples of the specialization of particular aspects of sensory systems to accomplish a highly specialized task (Endler, 1992). In the case of communication, sensory tuning for sender-receiver matching has been shown repeatedly. However, the converse may also be true. Overspecialization may come at the cost of reduced sensitivity to more general environmental signals. In such a case, it may be more beneficial to favor sensory generalization over specializa- tion in animals that engage in social behaviors less often than their more gregarious relatives do. Indeed, there are several examples of peripheral sender-receiver mismatching that may be explained by gains in sensitivity to prey or predator signals too (Mason, 1991; Römer, 2016). While we see in $A$. leptorhynchus dedicated codes for communication signals with distinct meaning and in distinct contexts (Marsat and Maler, 2010; Allen and Marsat, 2018), maintaining that level of specificity for conspecific communication may be costly both metabolically (Niven and Laughlin, 2008) and in regard to detecting environmental stimuli apart from communication signals.

In $A$. albifrons, we show a general match between signal characteristics, low-frequency chirp envelopes, and CNS sensitivity to low-frequency signals, but a lack of detailed coding that would allow for efficient discrimination of chirp identity in all contexts. Additional changes that would lead to a more accurate chirp coding could come at the expense of the ability to encode other stimuli. Therefore, for this species, limiting the resources dedicated to the coding of social signals in certain contexts (e.g., aggressive encounters with a fish with a high difference in EOD frequency) may allow the system to preserve or enhance sensitivity to other stimuli.

\section{References}

Allen KM, Marsat G (2018) Task-specific sensory coding strategies matched to detection and discrimination behaviors in Apteronotus leptorhynchus. J Exp Biol 221:jeb170563.

Aumentado-Armstrong T, Metzen MG, Sproule MKJ, Chacron MJ (2015) Electrosensory midbrain neurons display feature invariant responses to natural communication stimuli. PLoS Comput Biol 11:1-28.

Avila-Akerberg O, Krahe R, Chacron MJ (2010) Neural heterogeneities and stimulus properties affect burst coding in vivo. Neuroscience 168:300-313.

Bastian J (1981) Electrolocation. II. The effects of moving objects and other electrical stimuli on the activities of two categories of posterior lateral line lobe cells in Apteronotus albifrons. J Comp Physiol 144:481-494.

Bastian J (1986) Gain control in the electrosensory system: a role for the descending projections to the electrosensory lateral line lobe. J Comp Physiol A 158:505-515.

Bastian J, Chacron MJ, Maler L (2004) Plastic and nonplastic pyramidal cells perform unique roles in a network capable of adaptive redundancy reduction. Neuron 41:767-779.

Benda J, Longtin A, Maler L (2005) Spike-frequency adaptation separates transient communication signals from background oscillations. J Neurosci 25:2312-2321.

Benda J, Longtin A, Maler L (2006) A synchronizationdesynchronization code for natural communication signals. Neuron 52:347-358.

Borst A, Theunissen FE (1999) Information theory and neural coding. Nat Neurosci 2:947-957.

Bradbury JW, Vehrencamp SL (2011) Principles of animal communication, Ed 2. Sunderland, MA: Sinauer Associates.

Brumm H, Slabbekoorn H (2005) Acoustic communication in noise. Adv Study Behav 35:151-209.

Chacron MJ (2006) Nonlinear information processing in a model sensory system. J Neurophysiol 95:2933-2946.

Chacron MJ, Bastian J (2008) Population coding by electrosensory neurons. J Neurophysiol 99:1825-1835.

Chacron MJ, Maler L, Bastian J (2005) Feedback and feedforward control of frequency tuning to naturalistic stimuli. J Neurosci 25: 5521-5532. 
Creutzig F, Wohlgemuth S, Stumpner A, Benda J, Ronacher B, Herz AVM (2009) Timescale-invariant representation of acoustic communication signals by a bursting neuron. J Neurosci 29:25752580.

Deemyad T, Metzen MG, Pan Y, Chacron MJ (2013) Serotonin selectively enhances perception and sensory neural responses to stimuli generated by same-sex conspecifics. Proc Natl Acad Sci U S A 110:19609-19614.

Dunlap KD, Larkins-Ford J (2003) Diversity in the structure of electrocommunication signals within a genus of electric fish, Apteronotus. J Comp Physiol A 189:153-161.

Dunlap KD, Thomas P, Zakon HH (1998) Diversity of sexual dimorphism in electrocommunication signals and its androgen regulation in a genus of electric fish, Apteronotus. J Comp Physiol A 183:77-86.

Endler JA (1992) Signals, signal conditions, and the direction of evolution. Am Nat 139:S125-S153.

Frank K, Becker MC (1964) Microelectrodes for recording and stimulation In: Physical techniques in biological research (Nastuk WL, ed), pp 23-84. New York: Academic.

Fujimoto H, Hasegawa T, Watanabe D (2011) Neural coding of syntactic structure in learned vocalizations in the songbird. J Neurosci 31:10023.

Gabbiani F, Metzner W, Wessel R, Koch C (1996) From stimulus encoding to feature extraction in weakly electric fish. Nature 384 : 564-567.

Gerhardt HC, Schwartz JJ (2001) Auditory tuning and frequency preferences in Anuran. In: Anuran communication (Ryan M J, ed), pp. 73-85. Smithsonian Institution Press, Washington, DC.

Goldberg JM, Brown PB (1969) Response of binaural neurons of dog superior olivary complex to dichotic tonal stimuli: some physiological mechanisms of sound localization. J Neurophysiol 32:613636.

Hagedorn M, Heiligenberg W (1985) Court and spark: electric signals in the courtship and mating of gymnotoid fish. Anim Behav 33: 254-265.

Harris-Warrick RM, Marder E (1991) Modulation of neural networks for behavior. Annu Rev Neurosci 14:39-57.

Henninger J, Krahe R, Kirschbaum F, Grewe J, Benda J (2018) Statistics of natural communication signals observed in the wild identify important yet neglected stimulus regimes in weakly electric fish. J Neurosci 38:5456-5465.

Hofmann V, Chacron MJ (2017) Differential receptive field organizations give rise to nearly identical neural correlations across three parallel sensory maps in weakly electric fish. PLoS Comput Biol 13:e1005716

Houghton C, Sen K (2008) A new multineuron spike train metric. Neural Comput 20:1495-1511.

Hupé GJ (2012) Electrocommunication in a species of weakly electric fish apteronotus leptorhynchus: signal patterning and behaviour. Ottowa, ON, Canada: University of Ottawa.

Hupé GJ, Lewis JE (2008) Electrocommunication signals in free swimming brown ghost knifefish, Apteronotus leptorhynchus. J Exp Biol 211:1657-1667.

Itatani N, Klump GM (2014) Neural correlates of auditory streaming in an objective behavioral task. Proc Natl Acad Sci U S A 111:1073810743.

Kolodziejski JA, Sanford SE, Smith GT (2007) Stimulus frequency differentially affects chirping in two species of weakly electric fish: implications for the evolution of signal structure and function. J Exp Biol 210:2501-2509.

Krahe R, Bastian J, Chacron MJ (2008) Temporal processing across multiple topographic maps in the electrosensory system. J Neurophysiol 100:852-867.

Larson E, Billimoria CP, Sen K (2009) A biologically plausible computational model for auditory object recognition. J Neurophysiol 101:323-331.

Larson E, Perrone BP, Sen K, Billimoria CP (2010) A robust and biologically plausible spike pattern recognition network. J Neurosci 30:15566-15572.
Machens CK, Schütze H, Franz A, Kolesnikova O, Stemmler MB, Ronacher B, Herz AV (2003) Single auditory neurons rapidly discriminate conspecific communication signals. Nat Neurosci 6:341342.

Maler L, Sas E, Johnston S, Ellis W (1991) An atlas of the brain of the electric fish Apteronotus leptorhynchus. J Chem Neuroanat 4:138

Marsat G, Maler L (2010) Neural heterogeneity and efficient population codes for communication signals. J Neurophysiol 104:25432555.

Marsat G, Pollack GS (2004) Differential temporal coding of rhythmically diverse acoustic signals by a single interneuron. J Neurophysiol 92:939-48.

Marsat G, Proville RD, Maler L (2009) Transient signals trigger synchronous bursts in an identified population of neurons. J Neurophysiol 102:714-723.

Marsat G, Longtin L, Maler L (2012) Cellular and circuit properties supporting different sensory coding strategies in electric fish and other systems. Curr Opin Neurobiol 22:686-692.

Martinez-Conde S, Macknik SL, Hubel DH (2002) The function of bursts of spikes during visual fixation in the awake primate lateral geniculate nucleus and primary visual cortex. Proc Natl Acad Sci U S A 99:13920-13925.

Martinez D, Metzen MG, Chacron MJ (2016) Electrosensory processing in Apteronotus albifrons: implications for general and specific neural coding strategies across wave-type weakly electric fish species. J Neurophysiol 116:2909-2921.

Mason AC (1991) Hearing in a primitive ensiferan: the auditory system of Cyphoderris monstrosa (Orthoptera: Haglidae). J Comp Physiol A 168:351-363.

Metzen MG, Huang CG, Chacron MJ (2018) Descending pathways generate perception of and neural responses to weak sensory input. PLoS Biol 16:e2005239.

Metzner W (1999) Neural circuitry for communication and jamming avoidance in gymnotiform electric fish. J Exp Biol 202:1365-1375.

Metzner W, Heiligenberg W (1991) The coding of signals in the electric communication of the gymnotiform fish Eigenmannia: from electroreceptors to neurons in the torus semicircularis of the midbrain. J Comp Physiol A 169:135-150.

Metzner W, Juranek J (1997) A sensory brain map for each behavior? Proc Natl Acad Sci U S A 94:14798-14803.

Metzner W, Viete S (1996) The neuronal basis of communication and orientation in the weakly electric fish, Eigenmannia. I. Communication behavior or seeking a conspecific response. Naturwissenschaften 83:6-14.

Middleton JW, Longtin A, Benda J, Maler L (2006) The cellular basis for parallel neural transmission of a high-frequency stimulus and its low-frequency envelope. Proc Natl Acad Sci U S A 103:14596601.

Mouterde SC, Elie JE, Mathevon N, Theunissen FE (2017) Single neurons in the avian auditory cortex encode individual identity and propagation distance in naturally degraded communication calls. J Neurosci 37:3491-3510.

Neuhofer D, Ronacher B (2012) Influence of different envelope maskers on signal recognition and neuronal representation in the auditory system of a grasshopper. PLoS One 7:e34384.

Neuhofer D, Wohlgemuth S, Stumpner A, Ronacher B (2008) Evolutionarily conserved coding properties of auditory neurons across grasshopper species. Proc R Soc Lond B Biol Sci 275:1965-1974.

Niven JE, Laughlin SB (2008) Energy limitation as a selective pressure on the evolution of sensory systems. J Exp Biol 211:17921804.

Oswald A-MM, Chacron MJ, Doiron B, Bastian J, Maler L (2004) Parallel processing of sensory input by bursts and isolated spikes. J Neurosci 24:4351-4362.

Petzold JM, Alves-Gomes JA, Smith GT (2018) Chirping and asymmetric jamming avoidance responses in the electric fish Distocyclus conirostris. J Exp Biol 221:jeb178913. 
Petzold JM, Marsat G, Smith GT (2016) Co-adaptation of electric organ discharges and chirps in South American ghost knifefishes (Apteronotidae). J Physiol Paris 110:200-215.

Römer H (2016) Matched filters in insect audition: tuning curves and beyond. In: The ecology of animal senses: matched filters for economical sensing (von der Emde G, Warrant E, eds), pp 83-109. Cham, Germany: Springer International.

Saunders J, Bastian J (1984) The physiology and morphology of two types of electrosensory neurons in the weakly electric fish Apteronotus leptorhynchus. J Comp Physiol A 154:199-209.

Savard M, Krahe R, Chacron MJ (2011) Neural heterogeneities influence envelope and temporal coding at the sensory periphery. Neuroscience 172:270-284.

Schul J, Bush SL (2002) Non-parallel coevolution of sender and receiver in the acoustic communication system of treefrogs. Proc R Soc Lond B Biol Sci 269:1847-1852.

Smith AR, Proffitt MR, Ho WW, Mullaney CB, Maldonado-Ocampo JA, Lovejoy NR, Alves-Gomes JA, Smith GT (2016) Evolution of electric communication signals in the South American ghost knifefishes (Gymnotiformes: Apteronotidae): a phylogenetic comparative study using a sequence-based phylogeny. J Physiol 110:302-313.

Smith GT (2013) Evolution and hormonal regulation of sex differences in the electrocommunication behavior of ghost knifefishes (Apteronotidae). J Exp Biol 216:2421-2433.

Stamper SA, Carrera-G E, Tan EW, Fugère V, Krahe R, Fortune ES (2010) Species differences in group size and electrosensory interference in weakly electric fishes: Implications for electrosensory processing. Behav Brain Res 207:368-376.

Stamper SA, Fortune ES, Chacron MJ (2013) Perception and coding of envelopes in weakly electric fishes. J Exp Biol 216:2393-2402.
Stöckl A, Sinz F, Benda J, Grewe J (2014) Encoding of social signals in all three electrosensory pathways of Eigenmannia virescens. $J$ Neurophysiol 112:2076-2091.

Tootoonian S, Coen P, Kawai R, Murthy M (2012) Neural representations of courtship song in the Drosophila brain. J Neurosci 32:787-798.

Turner CR, Derylo M, de Santana CD, Alves-Gomes J a, Smith GT (2007) Phylogenetic comparative analysis of electric communication signals in ghost knifefishes (Gymnotiformes: Apteronotidae). J Exp Biol 210:4104-4122.

van Rossum MC (2001) A novel spike distance. Neural Comput 13:751-763.

Walz H, Grewe J, Benda J (2014) Static frequency tuning accounts for changes in neural synchrony evoked by transient communication signals. J Neurophysiol 112:752-765.

Woolley SMN, Moore JM (2011) Coevolution in communication senders and receivers: vocal behavior and auditory processing in multiple songbird species. Ann N Y Acad Sci 1225:155-165.

Zakon HH, Dunlap KD (1999) Sex steroids and communication signals in electric fish: a tale of two species. Brain Behav Evol 54:61-69.

Zakon HH, Smith GT (2002) Weakly electric fish: behavior, neurobiology, and neuroendocrinology. Horm Brain Behav 3: 349-375.

Zupanc GKH, Maler L (1993) Evoked chirping in the weakly electric fish Apteronotus leptorhynchus: a quantitative biophysical analysis. Can J Zool 71:2301-2310.

Zupanc GKH, Sîrbulescu RF, Nichols A, Ilies I (2006) Electric interactions through chirping behavior in the weakly electric fish, Apteronotus leptorhynchus. J Comp Physiol A Neuroethol Sens Neural Behav Physiol 192:159-173. 